Situations de handicap, fragilités sociales et rapport au territoire urbain

Disability, social fragility and urban space

Behinderung, soziale Fragilität und städtischer Raum

Couverture: Art inclusif: artistes handicapés au festival culturel de Wildwuchs à Bâle

Inclusive Art: disabled artists at Wildwuchs cultural festival in Basel

Inklusive Kunst: behinderte Künstler und Künstlerinnen am Basler Kulturfestival Wildwuchs

Foto: DoMINIK LABHARDT 


\section{Situations de handicap, fragilités sociales et rapport au territoire urbain Introduction au cahier thématique}

\section{Jean-Bernard Racine, Lausanne}

La géographie comme étude des conditions de possibilité de l'humanité, «le handicap comme une possibilité de l'humanité»! Une condition que notre société a toutes les peines du monde à concevoir d'une part, à en accepter les conséquences d'autre part. C'est ainsi que ce numéro de Geographica Helvetica a pour objectif de contribuer à une prise de conscience du handicap, tout en permettant d'imaginer les bases possibles d'une série d'approches réflexives, critiques et stratégiques, grâce à la participation de personnes engagées dans une réflexion plus générale sur les rapports de nos sociétés au territoire, qu'ils soient géographes, sociologues, urbanistes, juristes, spécialistes de la communication ou tout simplement activement engagés dans l'action ou la communication sur le terrain. Les uns et les autres partagent une même préoccupation: contribuer à faciliter la vie des «personnes en situation de handicap» comme on dit au Québec (Fougeyrollas 2001), ou comme d'autres préfèrent les qualifier, des «personnes ayant des incapacités». A l'heure du politiquement correct, il semblerait que plus personne n'ait encore le droit de parler tout simplement de «handicapés».

Compte tenu de la place disponible, il n'était pas possible de demander aux différents chercheurs mobilisés de se livrer chacun à un long travail sémantique sur la notion de handicap, singulièrement en ce qui concerne la mobilisation de certains concepts, d'ailleurs fort relativisés aujourd'hui au fur et à mesure de la prise de conscience du poids des représentations sociales du handicap et de la forte composante émotionnelle du problème posé par le «processus de production du handicap» au sein d'une société soucieuse de durabilité sociale (BoRIoli \& LAUB 2007). Editeurs, éditeur invité et soussigné, en même temps que les évaluateurs anonymes associés à notre revue ont pris leurs responsabilités en contrôlant le choix des articles retenus, espérant que ce numéro très particulier rencontrera les préoccupations d'un public largement ouvert à ce problème clé de notre temps. De fait, il concerne nos populations dans des proportions allant bien au-delà des seuls «pourcents» de personnes souffrant d'une incapacité.

On notera par ailleurs que dans une thématique d'une telle importance pour la société, il n'est évidemment pas possible de présenter un champ de connaissan- ces en se contentant de mobiliser les seules recherches dotées d'un label scientifique et universitaire. Ce numéro spécial de la revue a donc été ouvert à des personnes directement engagées dans l'action au niveau pratique.

De fait, la thématique du handicap s'est progressivement imposée ces dernières années comme l'une des dimensions critiques de toute réflexion sur l'organisation de l'espace urbain et les rapports qu'elle entretient avec les pratiques spatiales et sociales. Il s'agit d'une dimension «limite» à tous les sens du mot, dont cette collection d'articles ne saurait épuiser la description tant sont variées les formes d'incapacités rencontrées (motrices, sensorielles, mentales, psychiques). De même, on notera la grande variété des domaines où interviennent les obstacles à la transparence de l'espace urbain tel qu'il est pratiqué par les personnes dites «normales» (portes, trottoirs, automates, transports publics), autant d'obstacles physiques mais aussi sociaux liés aux représentations comme aux affects et aux émotions, pouvant faciliter ou compliquer la vie des usagers, mais également celle de ceux qui les accompagnent (parents, amis, soignants, professionnels ou bénévoles).

Coordinateur et éditeur en son temps de l'important ouvrage interdisciplinaire consacré au handicap, et joliment intitulé «Handicap: de la différence à la singularité. Enjeux au quotidien», publié par les Editions Médecine et Hygiène en 2007, le géographe lausannois JASON Borioli offre une réflexion sur le rôle que la société peut exercer dans les situations précaires auxquelles les personnes handicapées sont confrontées à l'heure actuelle. Il s'interroge sur les implications, les pratiques et les idéologies d'une remise en question de la «normalité» et fait le point sur l'évolution des études du handicap en géographie. Tout en sachant s'appuyer sur l'observation participante de «fragments de vie urbaine» propres à des personnes en situation de handicap, en même temps que sur une réflexion d'ordre sémantique sur l'évolution et l'enrichissement des représentations liées au phénomène du handicap dans nos sociétés - ne serait-ce qu'au niveau des acceptions du handicap telles que révélées par l'évolution du mot et de son intégration conceptuelle des origines à nos jours -, l'auteur présente les principaux modèles et théories issus des Disability Studies en accordant une attention particulière aux théories dites des «affordances» et de la «liminalité». Celles-ci semblent en effet offrir un terreau fertile à l'investigation géographique 
des «situations du handicap», concept important appelant à considérer que le handicap résulte de l'interaction entre l'individu, avec ses éventuelles déficiences et incapacités, et cet «environnement» qui peut présenter des obstacles ou des facilitateurs à l'accomplissement de ses activités quotidiennes. En d'autres termes, un problème de participation sociale plutôt que d'intégrité corporelle ou psychique.

De leur côté, partant d'un autre point de vue, les ingénieurs en environnement urbain de l'Institut national des sciences appliquées de Lyon, JeAN-Yves TousSAINT et SOPHIE VAREILLES racontent comment ils ont «rejoint» la problématique du handicap à travers la mobilisation des objets et leur assemblage en dispositifs techniques et spatiaux constituant les espaces publics urbains dans les activités sociales urbaines. Issue d'une recherche appliquée commandée par la Communauté urbaine du Grand Lyon, cherchant à évaluer des mobiliers urbains spécifiquement conçus et réalisés pour les publics aveugles et malvoyants, cette recherche précise et pointue, très focalisée, débouche néanmoins sur des énoncés aussi originaux que d'intérêt tout à fait général quant à la place de l'autonomie dans les comportements individuels et collectifs au sein de l'espace public. Les auteurs y précisent alors les conditions de sa reconquête et le rôle que peut jouer la technique dans les fonctions corporelles et cognitives. Ceci donne également l'occasion d'une étonnante rencontre avec les préoccupations tant techniques qu'éthiques et philosophiques liées aux effets de la privation des objets du monde quotidien sur la convivialité urbaine.

Animée par StÉPhanie Weiss, Matthias Drilling et DANiel Blumer, la présentation par l'équipe de sociologues bâlois du passage progressif des préoccupations du design dit universel tel qu'orienté sur les solutions à apporter, à l'accessibilité pour personnes ayant un handicap, à une accessibilité généralisée, est significative d'une évolution bienvenue des préoccupations. Elle est propre à la généralisation de la volonté de durabilité sociale en matière de développement urbain. Celle-ci repose notamment sur une pratique et une volonté politique essentielles: la participation et la diversité. Les exemples proposés en matière d'habitat montrent bien l'intérêt d'un mode de planification associant simultanément produit et solution, et donc, in fine, l'«urbanité», au bénéfice de l'ensemble des résidents.

C'est dans le même esprit que les géographes lausannois Christophe Mager et Laurent MatThey élargissent l'ensemble des préoccupations de ce numéro et légitiment son titre général, «Situations de handicap, fragilités sociales et rapport au territoire urbain». Reconnaissant dans la morphologie de l'espace urbain un agent des processus de différenciation en même temps qu'elle assure l'inertie de la morphologie sociale, les auteurs prennent acte de la montée de la précarité dans les centres urbains en Suisse. Dans le cadre d'une réflexion théorique remarquablement pointue, ils rappellent les mécanismes économiques, institutionnels et les effets de lieux qui la fondent. En s'appuyant sur des données d'enquêtes réalisées depuis 2004 à l'Observatoire de la ville et du développement durable de l'Université de Lausanne, ils illustrent l'accroissement de la précarité sous ses diverses formes, présentent quelques-uns des tenants et aboutissants de la fragilisation et de la dualisation des espaces métropolitains et tentent d'expliciter les modalités émergentes d'accompagnement des usages fragiles de la ville sur lesquels finalement leur article se focalise. Les auteurs évoquent alors les différents dispositifs de lutte contre la pauvreté, l'exclusion et la précarisation de l'emploi, les politiques d'intégration des populations migrantes, les opérations de prévention des violences ou des addictions qui constituent en effet des axes de structuration majeurs des agendas municipaux. Il s'agit là d'une manière d'en appeler, de façon stimulante, à l'invention de modalités nouvelles d'intervention et de gouvernance. Point fort des développements sociaux qu'il est possible d'observer dans les villes suisses: l'occasion d'un appel à «repenser la citoyenneté urbaine».

Restent enfin les deux articles proposés sous la rubrique «contributions appliquées» par Martin HaUG d'une part et Carine Bonsack et Monique Richoz d'autre part, personnalités directement engagées dans l'action, la prévention, la correction et, heureusement, l'imagination. Il s'agit certes de contributions «appliquées» qui ne relèvent pas comme telles de contributions habituellement écrites pour une revue scientifique, mais c'est bien l'éditeur invité et soussigné d'une revue scientifique qui a fait appel à l'expérience tout à fait unique de ces auteurs, en considérant cet apport comme étant de grande valeur, documentaire certes, mais également stratégique. On les considérera donc d'expression de «bonnes pratiques». Ces deux contributions se situent «dans la vie», rappelant et illustrant certains prolongements des tenants et aboutissants d'une géographie réellement «active». C'est justement en cherchant, comme géographe, à agir «dans la vie», que l'éditeur invité a rencontré ces «spécialistes» engagés dans l'action. Une occasion unique de mieux comprendre le handicap en vue de la prise en compte des besoins de chacun.

Chargé de l'intégration et de l'égalité des personnes handicapées du canton de Bâle-Ville Martin Haug montre comment lutter contre la ségrégation et la stigmatisation, de même qu'il expose les bases juridiques susceptibles de promouvoir la participation des personnes handicapées dans la vie publique. A l'évidence, 
qu'il s'agisse de définir des projets ou de mobiliser des ressources, la part de l'invention et de la créativité au sein des structures administratives cantonales représente un sérieux défi, tant intellectuel que moral. Il semble remarquablement relevé dans le canton de Bâle-Ville.

Il en va de même, dans le cadre de responsabilités réalisées à Lausanne, à l'échelle plus étroitement urbaine et dans le domaine culturel. C'est ce que montre l'expérience renouvelée de l'Accès-Cible telle que promue par l'association de la Nuit des Musées lausannois pour faciliter l'accès à la culture en faveur des personnes handicapées et que nous présentent CARINE BonsACK, spécialiste en communication, et MoNique Richoz, directrice de Pro Infirmis Vaud. L'expérience de l'Accès-Cible représente en la matière un excellent exemple de pratique également créative en matière d'accessibilité des personnes en situation de handicap au domaine culturel et éventuellement au partage social du sens et des émotions. Corrélativement, ce type de rencontre permet également de sensibiliser le grand public à l'intégration et à la participation sociale des personnes ayant une déficience, avec cet atout supplémentaire que ces diverses actions et mesures sont conçues et réalisées en partenariat étroit avec les «parties prenantes» que sont les personnes en situation d'handicap. Ce genre d'initiative donne matière à réflexion et, espère-t-on, à motivation pour une géographie active et volontaire, engagée dans une forme de recherche-action.

Le soussigné avait déjà eu l'occasion de s'exprimer sur ce thème au sein de l'ouvrage édité en 2007 sous la direction de JASON BorIOLI et RAPHAËL LAUB, ses étudiants. On lui permettra de répéter ici ce qu'il avait cru essentiel de partager avec la géographie d'aujourd'hui:

«(..) l'espace vécu par les personnes en situation de handicap interpelle tout à la fois son sens de l'humain et sa volonté de contribuer à humaniser leur espace de vie, singulièrement au niveau de l'accès à une mobilité plus généralisée, condition aujourd'hui, dans un univers urbain de plus en plus fragmenté, d'une socialisation au moins satisfaisante» (RACINE 2007:119).

\section{Bibliographie}

Borioli, J. \& R. Laub (2007): Des acceptions du handicap. L'évolution du mot «handicap» de ses origines à nos jours. - In: Borioli, J. \& R. LAUB (éds): Handicap: de la différence à la singularité. Enjeux au quotidien. - Chêne-Bourg: Editions Médecine et Hygiène: 25-45. Fougeyrollas, P. (2001): Le processus de production du handicap: l'expérience québécoise. - In: DE RIEDMATTEN, R. (éd.): Une nouvelle approche de la différence. Comment repenser le «handicap». - Genève: Editions Médecine et Hygiène: 101-122.

Racine, J.-B. (2007): Situations de handicaps et rapports au territoire. - In: BoRIoLI, J. \& R. LAUB (éds): Handicap: de la différence à la singularité. Enjeux au quotidien. - Chêne-Bourg: Editions Médecine et Hygiène: 119-133.

Prof. Dr. Jean-Bernard Racine, (Université de Lausanne), Av. du Grey 16, CH-1004 Lausanne, Suisse. e-mail: jean-bernard.racine@unil.ch 